\title{
On permutations of order dividing a given integer
}

\author{
Alice C. Niemeyer • Cheryl E. Praeger
}

Received: 31 March 2006 / Accepted: 2 January 2007 /

Published online: 17 April 2007

(C) Springer Science+Business Media, LLC 2007

\begin{abstract}
We give a detailed analysis of the proportion of elements in the symmetric group on $n$ points whose order divides $m$, for $n$ sufficiently large and $m \geq n$ with $m=O(n)$.
\end{abstract}

Keywords Symmetric group · Proportions

Mathematics Subject Classification (2000) Primary 20B30 · Secondary 20P05

\section{Introduction}

The study of orders of elements in finite symmetric groups goes back at least to the work of Landau [7, p. 222] who proved that the maximum order of an element of the symmetric group $S_{n}$ on $n$ points is $e^{(1+o(1))(n \log n)^{1 / 2}}$. Erdôs and Turán took a probabilistic approach in their seminal work in the area, proving in $[4,5]$ that, for a uniformly distributed random element $g \in S_{n}$, the random variable $\log |g|$ is normally distributed with mean $(1 / 2) \log ^{2} n$ and standard deviation $\frac{1}{\sqrt{3}} \log ^{3 / 2}(n)$. Thus most permutations in $S_{n}$ have order considerably larger than $O(n)$. Nevertheless, permutations of order $O(n)$, that is, of order at most $c n$ for some constant $c$, have received some attention in the literature. Let $P(n, m)$ denote the proportion of permutations $g \in S_{n}$ which satisfy $g^{m}=1$, that is to say, $|g|$ divides $m$. In 1952 Chowla, Herstein and Scott [3] found a generating function and some recurrence relations for $P(n, m)$ for $m$ fixed, and asked for its asymptotic behaviour for large $n$. Several years later,

A.C. Niemeyer $(\bowtie) \cdot$ C.E. Praeger

School of Mathematics and Statistics, University of Western Australia, Nedlands, WA 6907,

Australia

e-mail: alice@maths.uwa.edu.au

C.E. Praeger

e-mail: praeger@maths.uwa.edu.au 
Moser and Wyman [10, 11] derived an asymptotic for $P(n, m)$, for a fixed prime number $m$, expressing it as a contour integral. Then in 1986, Wilf [17] obtained explicitly the limiting value of $P(n, m)$ for an arbitrary fixed value of $m$ as $n \rightarrow \infty$, see also the paper [15] of Volynets. Other authors have considered equations $g^{m}=h$, for a fixed integer $m$ and $h \in S_{n}$, see [2, 6, 8, 9].

However in many applications, for example in [1], the parameters $n$ and $m$ are linearly related, so that $m$ is unbounded as $n$ increases. For the special case where $m=n$, Warlimont [16] showed in 1978 that most elements $g \in S_{n}$ satisfying $g^{n}=1$ are $n$-cycles, namely he proved that $P(n, n)$, for $n$ sufficiently large, satisfies

$$
\frac{1}{n}+\frac{2 c}{n^{2}} \leq P(n, n) \leq \frac{1}{n}+\frac{2 c}{n^{2}}+O\left(\frac{1}{n^{3-o(1)}}\right)
$$

where $c=1$ if $n$ is even and $c=0$ if $n$ is odd. Note that the proportion of $n$-cycles in $S_{n}$ is $1 / n$ and, if $n$ is even, the proportion of elements that are a product of two cycles of length $n / 2$ is $2 / n^{2}$. Warlimont's result proves in particular that most permutations satisfying $g^{n}=1$ are $n$-cycles. More precisely it implies that the conditional probability that a random element $g \in S_{n}$ is an $n$-cycle, given that $g^{n}=1$, lies between $1-2 c n^{-1}-O\left(n^{-2+o(1)}\right)$ and $1-2 c n^{-1}+O\left(n^{-2}\right)$.

The main results of this paper, Theorems 1.1 and 1.2, generalise Warlimont's result, giving a detailed analysis of $P(n, m)$ for large $n$, where $m=O(n)$ and $m \geq n$. For this range of values of $n$ and $m$, we have $r n \leq m<(r+1) n$ for some positive integer $r$, and we analyse $P(n, m)$ for $m$ in this range, for a fixed value of $r$ and $n \rightarrow \infty$. It turns out that the kinds of elements that make the largest contribution to $P(n, m)$ depend heavily on the arithmetic nature of $m$, for example, on whether $m$ is divisible by $n$ or by $r+1$. We separate out several cases in the statement of our results. Theorem 1.1 deals with two cases for which we give asymptotic expressions for $P(n, m)$. The first of these reduces in the case $m=n$ to Warlimont's theorem [16] (modulo a small discrepancy in the error term). For other values of $m$ lying strictly between $r n$ and $(r+1) n$ we obtain in Theorem 1.2 only an upper bound for $P(n, m)$, since the exact value depends on both the arithmetic nature and the size of $m$ (see also Remark 1.3).

Theorem 1.1 Let $n$ and $r$ be positive integers. Then for a fixed value of $r$ and sufficiently large $n$, the following hold.

(a) $P(n, r n)=\frac{1}{n}+\frac{c(r)}{n^{2}}+O\left(\frac{1}{n^{2.5-o(1)}}\right)$ where $c(r)=\sum\left(1+\frac{i+j}{2 r}\right)$ and the sum is over all pairs $(i, j)$ such that $1 \leq i, j \leq r^{2}, i j=r^{2}$, and both $r+i, r+j$ divide $r n$. In particular $c(1)=0$ if $n$ is odd, and 2 if $n$ is even.

(b) If $r=t !-1$ and $m=t !(n-t)=(r+1) n-t \cdot t$ !, then

$$
P(n, m)=\frac{1}{n}+\frac{t+c^{\prime}(r)}{n^{2}}+O\left(\frac{1}{n^{2.5-o(1)}}\right)
$$

where $c^{\prime}(r)=\sum\left(1+\frac{i+j-2}{2(r+1)}\right)$ and the sum is over all pairs $(i, j)$ such that $1<$ $i, j \leq(r+1)^{2},(i-1)(j-1)=(r+1)^{2}$, and both $r+i, r+j$ divide $m$. 
Theorem 1.2 Let $n, m, r$ be positive integers such that $r n<m<(r+1) n$, and $\delta$ a real number such that $0<\delta \leq 1 / 4$. Then for a fixed value of $r$ and sufficiently large $n$,

$$
P(n, m) \leq \frac{\alpha \cdot(r+1)}{m}+\frac{k(r)}{n^{2}}+O\left(\frac{1}{n^{2.5-2 \delta}}\right)
$$

where $k(r)=\frac{4(r+3)^{4}}{r^{2}}$ and

$$
\alpha= \begin{cases}1 & \text { if } r+1 \text { divides } m \text { and } n-\frac{m}{r+1}<\frac{m}{2(r+1)(r+2)-1} \\ 0 & \text { otherwise. }\end{cases}
$$

Remark 1.3 (a) In Theorem 1.1(a), the leading term $1 / n$ is the proportion of $n$-cycles, while the proportion of permutations containing an $(n-t)$-cycle is $\frac{1}{n-t}=\frac{1}{n}+\frac{t}{n^{2}}+$ $O\left(\frac{1}{n^{3}}\right)$, which contributes to the first two terms in Theorem 1.1(b). The terms $\frac{c(r)}{n^{2}}$ and $\frac{c^{\prime}(r)}{n^{2}}$ correspond to permutations in $S_{n}$ that have two long cycles, and these have lengths $\frac{m}{r+i}$ and $\frac{m}{r+j}$, for some $(i, j)$ satisfying the conditions in Theorem 1.1 (a) or (b) respectively, (where $m=r n$ in part (a)).

(b) In Theorem 1.2, if $r+1$ divides $m$ and $n-m /(r+1)<\frac{m}{2(r+1)(r+2)-1}$, then the term $(r+1) / m$ comes from elements containing a cycle of length $m /(r+1)$. The term $\frac{k(r)}{n^{2}}$ corresponds to permutations with exactly two 'large' cycles. More details are given in Remark 3.4.

Our interest in $P(n, m)$ arose from algorithmic applications concerning finite symmetric groups. For example, $n$-cycles in $S_{n}$ satisfy the equation $g^{n}=1$, while elements whose cycle structure consists of a 2-cycle and a single additional cycle of odd length $n-t$, where $t=2$ or 3 , satisfy the equation $g^{2(n-t)}=1$. For an element $g$ of the latter type we can construct a transposition by forming the power $g^{n-t}$. In many cases the group $S_{n}$ is not given as a permutation group in its natural representation, and, while it is possible to test whether an element $g$ satisfies one of these equations, it is often impossible to determine its cycle structure with certainty. It is therefore important to have lower bounds on the conditional probability that a random element $g$ has a desired cycle structure, given that it satisfies an appropriate equation. Using Theorem 1.1, we obtained the following estimates of various conditional probabilities.

Corollary 1.4 Let $r$, $n$ be positive integers and let $g$ be a uniformly distributed random element of $S_{n}$. Then for a fixed value of $r$ and sufficiently large $n$, the following hold, where $c(r)$ and $c^{\prime}(r)$ are as in Theorem 1.1 .

(a) The conditional probability $P$ that $g$ is an $n$-cycle, given that $|g|$ divides $r n$, satisfies

$$
1-\frac{c(r)}{n}-O\left(\frac{1}{n^{1.5-o(1)}}\right) \leq P \leq 1-\frac{c(r)}{n}+O\left(\frac{1}{n^{2}}\right) .
$$


(b) If $r=t !-1$, then the conditional probability $P$ that $g$ contains an $(n-t)$-cycle, given that $|g|$ divides $t !(n-t)$, satisfies

$$
1-\frac{c^{\prime}(r)}{n}-O\left(\frac{1}{n^{1.5-o(1)}}\right) \leq P \leq 1-\frac{c^{\prime}(r)}{n}+O\left(\frac{1}{n^{2}}\right) .
$$

We note that Theorem 1.1 improves the upper bound of $(1+o(1)) / n$ obtained in [1, Theorem 3.7], while Corollary 1.4 improves the corresponding lower bound of $1-o(1)$ of [1, Theorem 1.3(a)]. These results have been developed and refined further in [13] to derive explicit 'non-asymptotic' bounds that hold for all $n$ and can be applied directly to improve the recognition algorithms for $S_{n}$ and $A_{n}$ in [1].

Commentary on our approach Warlimont's proof in [16] of an upper bound for $P(n, n)$ and the proof of [1, Theorem 3.7] by Beals and Seress of an upper bound for $P(n, m)$ for certain values of $m$, rely on dividing the elements of $S_{n}$ into disjoint unions of smaller sets. Warlimont divides the elements according to how many 'large' cycles a permutation contains. Fix a real number $s$ such that $1 / 2<s<1$. We say that a cycle of a permutation in $S_{n}$ is $s$-small if its length is strictly less than $n^{s}$, and is $s$-large otherwise. Beals and Seress divide the elements according to the number of cycles in which three specified points lie. Both strategies are sufficient to prove Warlimont's result or the slightly more general results of [1, Theorem 3.7]. However, neither is sufficient to prove the general results in this paper. In particular, Warlimont's approach breaks down when trying to estimate the proportion of elements with no or only one large cycle, which is perhaps why no progress has been made since his paper [16] towards answering Chowla, Herstein and Scott's original question about the asymptotic behaviour of $P(n, m)$ for large $n$. One of the key ideas that allowed us to generalise Warlimont's work is the insight that the number of permutations which contain no $s$-large cycles can be estimated by considering their behaviour on three specified points. Another important strategy is our careful analysis of elements containing only one large cycle by separating out divisors of $m$ which are very close to $n$.

We regard Theorem 1.5 below as the main outcome of the first stage of our analysis. It is used in the proof of Theorem 1.1 and is applied in further work of the authors in [12]. The statement of Theorem 1.5 involves the number $d(m)$ of positive divisors of $m$, and the fact that $d(m)=m^{o(1)}$, see Notation 2.1(c). It estimates the proportion $P_{0}(n, m)$ of elements of $S_{n}$ of order dividing $m$ and having no $s$-large cycles.

Theorem 1.5 Let $n, m$ be positive integers such that $m \geq n$, and let $s$ be a positive real number such that $1 / 2<s<1$. Then, with $P_{0}(n, m)$ as defined above, there is a constant $c$ such that

$$
P_{0}(n, m)<\frac{c d(m) m^{2 s}}{n^{3}}=O\left(\frac{m^{2 s+o(1)}}{n^{3}}\right) .
$$

Theorem 1.5 is proved in Section 2 and the other results are proved in Section 3. 


\section{Proof of Theorem 1.5}

In this section we introduce some notation that will be used throughout the paper, and we prove Theorem 1.5. Note that the order $|g|$ of a permutation $g \in S_{n}$ divides $m$ if and only if the length of each cycle of $g$ divides $m$. Thus $P(n, m)$ is the proportion of elements in $S_{n}$ all of whose cycle lengths divide $m$. As indicated in the introduction, we estimate $P(n, m)$ by partitioning this proportion in various ways. Sometimes the partition is according to the number of $s$-large cycle lengths, and at other times it is defined in terms of the cycles containing certain points. We specify these partitions, and give some other notation, below.

Notation 2.1 The numbers $n, m$ are positive integers, and the symmetric group $S_{n}$ acts naturally on the set $\Omega=\{1,2, \ldots, n\}$.

(a) $s$ is a real number such that $1 / 2<s<1$. A divisor $d$ of $m$ is said to be $s$-large or $s$-small if $d \geq m^{s}$ or $d<m^{s}$, respectively; $D_{\ell}$ and $D_{s}$ denote the sets of all $s$-large and $s$-small divisors $d$ of $m$, respectively, such that $d \leq n$.

(b) For $g \in S_{n}$ with order dividing $m$, a $g$-cycle of length $d$ is called $s$-large or $s$-small according as $d$ is an $s$-large or $s$-small divisor of $m$.

(c) $d(m)$ denotes the number of positive divisors of $m$ and $\delta$ and $c_{\delta}$ are positive real numbers such that $\delta<s$ and $d(m) \leq c_{\delta} m^{\delta}$ for all $m \in \mathbf{N}$.

(d) The following functions of $n$ and $m$ denote the proportions of elements $g \in S_{n}$ of order dividing $m$ and satisfying the additional properties given in the last column of the table below.

\begin{tabular}{ll}
\hline$P_{0}(n, m)$ & all $g$-cycles are $s$-small \\
$P_{0}^{(1)}(n, m)$ & all $g$-cycles are $s$-small and \\
& $1,2,3$ lie in the same $g$-cycle, \\
$P_{0}^{(2)}(n, m)$ & all $g$-cycles are $s$-small and \\
& $1,2,3$ lie in exactly two $g$-cycles \\
$P_{0}^{(3)}(n, m)$ & all $g$-cycles are $s$-small and \\
& $1,2,3$ lie in three different $g$-cycles \\
$P_{1}(n, m)$ & $g$ contains exactly one $s$-large cycle \\
$P_{2}(n, m)$ & $g$ contains exactly two $s$-large cycles \\
$P_{3}(n, m)$ & $g$ contains exactly three $s$-large cycles \\
$P_{\geq 4}(n, m)$ & $g$ contains at least four $s$-large cycles \\
\hline
\end{tabular}

With respect to part (c) we note, see [14, pp. 395-396], that for each $\delta>0$ there exists a constant $c_{\delta}>0$ such that $d(m) \leq c_{\delta} m^{\delta}$ for all $m \in \mathbf{N}$. This means that the parameter $\delta$ can be any positive real number and in particular that $d(m)=m^{o(1)}$. Note that

$$
P_{0}(n, m)=P_{0}^{(1)}(n, m)+P_{0}^{(2)}(n, m)+P_{0}^{(3)}(n, m)
$$

and

$$
P(n, m)=P_{0}(n, m)+P_{1}(n, m)+P_{2}(n, m)+P_{3}(n, m)+P_{\geq 4}(n, m) .
$$

We begin by deriving recursive expressions for the $P_{0}^{(i)}(n, m)$. 
Lemma 2.2 Using Notation 2.1, the following hold, where we take $P_{0}(0, m)=1$.

(a) $P_{0}^{(1)}(n, m)=\frac{(n-3) !}{n !} \sum_{d \in D_{s}, d \geq 3}(d-1)(d-2) P_{0}(n-d, m)$,

(b) $P_{0}^{(2)}(n, m)=\frac{3(n-3) !}{n !} \sum_{\substack{d_{1}, d_{2} \in D_{s} \\ 2 \leq d_{2}}}\left(d_{2}-1\right) P_{0}\left(n-d_{1}-d_{2}, m\right)$,

(c) $P_{0}^{(3)}(n, m)=\frac{(n-3) !}{n !} \sum_{\substack{2 \leq d_{2}, d_{1}+d_{2} \leq n \\ d_{1}, d_{2}, d_{3} \in D_{s} \\ d_{1}+d_{2}+d_{3} \leq n}} P_{0}\left(n-d_{1}-d_{2}-d_{3}, m\right)$.

Proof We first compute $P_{0}^{(1)}(n, m)$, the proportion of those permutations $g \in S_{n}$ of order dividing $m$ with all cycles $s$-small, for which the points $1,2,3$ are contained in one $g$-cycle, $C$ say, of length $d$ with $d \in D_{s}$ and $d \geq 3$. We can choose the remainder of the support set of $C$ in $\left(\begin{array}{l}n-3 \\ d-3\end{array}\right)$ ways and then the cycle $C$ in $(d-1)$ ! ways. The rest of the permutation $g$ can be chosen in $P_{0}(n-d, m)(n-d)$ ! ways. Thus, for a given $d$, the number of such elements is $(n-3) !(d-1)(d-2) P_{0}(n-d, m)$. We obtain the proportion $P_{0}^{(1)}(n, m)$ by summing over all $d \in D_{s}$ with $d \geq 3$, and then dividing by $n$ !, so part (a) is proved.

Next we determine the proportion $P_{0}^{(2)}(n, m)$ of those permutations $g \in S_{n}$ of order dividing $m$ with all cycles $s$-small, for which one of the points $1,2,3$ is contained in a $g$-cycle $C_{1}$, and the other two of these points are contained in a different $g$-cycle $C_{2}$. Let $d_{1}$ and $d_{2}$ denote the lengths of the cycles $C_{1}$ and $C_{2}$, respectively, so $d_{1}, d_{2} \in D_{s}$ and $d_{2} \geq 2$. Firstly we choose the support set of $C_{1}$ in $\left(\begin{array}{c}n-3 \\ d_{1}-1\end{array}\right)$ ways and the cycle $C_{1}$ in $\left(d_{1}-1\right)$ ! ways. Secondly we choose the support set of $C_{2}$ in $\left(\begin{array}{c}n-d_{1}-2 \\ d_{2}-2\end{array}\right)$ ways and the cycle $C_{2}$ in $\left(d_{2}-1\right)$ ! ways. Finally, the rest of the permutation $g$ is chosen in $P_{0}\left(n-d_{1}-d_{2}, m\right)\left(n-d_{1}-d_{2}\right)$ ! ways. Thus, for a given pair $d_{1}, d_{2}$, the number of these elements is $(n-3) !\left(d_{2}-1\right) P_{0}\left(n-d_{1}-d_{2}, m\right)$. Since there are three choices for $C_{1} \cap\{1,2,3\}$, we have

$$
P_{0}^{(2)}(n, m)=\frac{3(n-3) !}{n !} \sum_{\substack{d_{1}, d_{2} \in D_{s} \\ 2 \leq d_{2}, d_{1}+d_{2} \leq n}}\left(d_{2}-1\right) P_{0}\left(n-d_{1}-d_{2}, m\right) .
$$

Finally we consider the proportion $P_{0}^{(3)}(n, m)$ of those permutations $g \in S_{n}$ of order dividing $m$ with all cycles $s$-small, for which each one of the points $1,2,3$ is contained in a separate $g$-cycle, say $C_{i}$ contains $i$ and $C_{i}$ has length $d_{i} \in D_{s}$. We can choose, in order, the support set of $C_{1}$ in $\left(\begin{array}{c}n-3 \\ d_{1}-1\end{array}\right)$ ways and the cycle $C_{1}$ in $\left(d_{1}-1\right)$ ! ways, the support set of $C_{2}$ in $\left(\begin{array}{c}n-d_{1}-2 \\ d_{2}-1\end{array}\right)$ ways and the cycle $C_{2}$ in $\left(d_{2}-1\right)$ ! ways, the support set of $C_{3}$ in $\left(\begin{array}{c}n-d_{1}-d_{2}-1 \\ d_{3}-1\end{array}\right)$ ways and the cycle $C_{3}$ in $\left(d_{3}-1\right)$ ! ways, and the rest of the permutation in $P_{0}\left(n-d_{1}-d_{2}-d_{3}, m\right)\left(n-d_{1}-d_{2}-d_{3}\right)$ ! ways. The expression for $P_{0}^{(3)}(n, m)$ in part (c) now follows.

Next we derive expressions for the $P_{i}(n, m)$ and $P_{\geq 4}(n, m)$. 
Lemma 2.3 Using Notation 2.1, and writing $P_{0}(0, m)=1$,

(a) $P_{0}(n, m)=\frac{1}{n} \sum_{d \in D_{s}} P_{0}(n-d, m)$

(b) $P_{1}(n, m)=\sum_{d \in D_{\ell}} \frac{1}{d} P_{0}(n-d, m)$,

(c) $P_{2}(n, m)=\frac{1}{2} \sum_{d_{1}, d_{2} \in D_{\ell}} \frac{1}{d_{1} d_{2}} P_{0}\left(n-d_{1}-d_{2}, m\right)$, where the sum is over all ordered pairs $\left(d_{1}, d_{2}\right)$ with $d_{1}+d_{2} \leq n$.

(d) $P_{3}(n, m)=\frac{1}{6} \sum_{d_{1}, d_{2}, d_{3} \in D_{\ell}} \frac{1}{d_{1} d_{2} d_{3}} P_{0}\left(n-d_{1}-d_{2}-d_{3}, m\right)$, where the sum is over all ordered triples $\left(d_{1}, d_{2}, d_{3}\right)$ with $d_{1}+d_{2}+d_{3} \leq n$.

(e) $P_{\geq 4}(n, m) \leq \frac{1}{24} \sum_{d_{1}, d_{2}, d_{3}, d_{4} \in D_{\ell}} \frac{1}{d_{1} d_{2} d_{3} d_{4}} P\left(n-d_{1}-d_{2}-d_{3}-d_{4}, m\right)$, where the sum is over all ordered 4-tuples $\left(d_{1}, d_{2}, d_{3}, d_{4}\right)$ with $d_{1}+d_{2}+d_{3}+d_{4} \leq n$.

Proof For each permutation in $S_{n}$ of order dividing $m$ and all cycles $s$-small, the point 1 lies in a cycle of length $d$, for some $d \in D_{s}$. For this value of $d$ there are $\left(\begin{array}{l}n-1 \\ d-1\end{array}\right)(d-1)$ ! choices of $d$-cycles containing 1 , and $P_{0}(n-d, m)(n-d)$ ! choices for the rest of the permutation. Summing over all $d \in D_{s}$ yields part (a).

The proportion of permutations in $S_{n}$ of order dividing $m$ and having exactly one $s$-large cycle of length $d$ is $\left(\begin{array}{l}n \\ d\end{array}\right)(d-1) ! P_{0}(n-d, m)(n-d) ! / n !$. Summing over all $d \in D_{\ell}$ yields part (b).

In order to find the proportion of elements in $S_{n}$ of order dividing $m$ and having exactly two $s$-large cycles we count triples $\left(C_{1}, C_{2}, g\right)$, where $C_{1}$ and $C_{2}$ are cycles of lengths $d_{1}$ and $d_{2}$ respectively, $d_{1}, d_{2} \in D_{\ell}, g \in S_{n}$ has order dividing $m, g$ contains $C_{1}$ and $C_{2}$ in its disjoint cycle representation, and all other $g$-cycles are $s$-small. For a given $d_{1}, d_{2}$, we have $\left(\begin{array}{c}n \\ d_{1}\end{array}\right)\left(d_{1}-1\right)$ ! choices for $C_{1}$, then $\left(\begin{array}{c}n-d_{1} \\ d_{2}\end{array}\right)\left(d_{2}-1\right)$ ! choices for $C_{2}$, and then the rest of the element $g$ containing $C_{1}$ and $C_{2}$ can be chosen in $P_{0}\left(n-d_{1}-d_{2}, m\right)\left(n-d_{1}-d_{2}\right)$ ! ways. Thus the ordered pair $\left(d_{1}, d_{2}\right)$ contributes $\frac{n !}{d_{1} d_{2}} P_{0}\left(n-d_{1}-d_{2}, m\right)\left(n-d_{1}-d_{2}\right)$ ! triples, and each element $g$ with the properties required for part (c) contributes exactly two of these triples. Hence, summing over ordered pairs $d_{1}, d_{2} \in D_{\ell}$ yields (c).

Similar counts are used for parts (d) and (e). For $P_{3}(n, m), P_{\geq 4}(n, m)$ we count 4-tuples $\left(C_{1}, C_{2}, C_{3}, g\right)$ and 5-tuples $\left(C_{1}, C_{2}, C_{3}, C_{4}, g\right)$ respectively, such that, for each $i, C_{i}$ is a cycle of length $d_{i}$ for some $d_{i} \in D_{\ell}, g \in S_{n}$ has order dividing $m$, and $g$ contains all the cycles $C_{i}$ in its disjoint cycle representation. The reason we have an inequality for $P_{\geq 4}(n, m)$ is that in this case each $g$ occurring has at least four $s$-large cycles and hence occurs in at least 24 of the 5-tuples, but possibly more.

We complete this section by giving a proof of Theorem 1.5. The ideas for its proof were developed from arguments in Warlimont's paper [16]. 
Lemma 2.4 Let $m \geq n \geq 3$, and let $s, \delta$ be as in Notation 2.1. Then

$$
P_{0}(n, m)<\frac{\left(1+3 c_{\delta}+c_{\delta}^{2}\right) d(m) m^{2 s}}{n(n-1)(n-2)}<\frac{c^{\prime} d(m) m^{2 s}}{n^{3}}=O\left(\frac{m^{2 s+\delta}}{n^{3}}\right)
$$

where, if $n \geq 6$, we may take

$$
c^{\prime}=\left\{\begin{array}{ll}
2\left(1+3 c_{\delta}+c_{\delta}^{2}\right) & \text { for any } m \geq n \\
10 & \text { if } m \geq c_{\delta}^{1 /(s-\delta)}
\end{array} .\right.
$$

In particular Theorem 1.5 is true. Moreover, if in addition $n \geq m^{s}+c n^{a}$ for some positive constants $a, c$ with $a \leq 1$, then $P_{0}(n, m)=O\left(\frac{m^{2 s+2 \delta}}{n^{1+3 a}}\right)$.

Proof First assume only that $m \geq n \geq 3$. Let $D_{s}$, and $P_{0}^{(i)}(n, m)$, for $i=1,2,3$, be as in Notation 2.1. By $(1), P_{0}(n, m)$ is the sum of the $P_{0}^{(i)}(n, m)$. We first estimate $P_{0}^{(1)}(n, m)$. By Lemma 2.2(a), and using the fact that $d<m^{s}$ for all $d \in D_{s}$,

$$
P_{0}^{(1)}(n, m) \leq \frac{(n-3) !}{n !} \sum_{\substack{d \in D_{s} \\ d \geq 3}}(d-1)(d-2)<\frac{d(m) m^{2 s}}{n(n-1)(n-2)} .
$$

Similarly, by Lemma 2.2(b),

$$
P_{0}^{(2)}(n, m)<\frac{3(n-3) !}{n !} \sum_{d_{1}, d_{2} \in D_{s}}\left(d_{2}-1\right) \leq \frac{3 d(m)^{2} m^{s}}{n(n-1)(n-2)}
$$

and by Lemma 2.2(c),

$$
P_{0}^{(3)}(n, m)<\frac{(n-3) !}{n !} \sum_{d_{1}, d_{2}, d_{3} \in D_{s}} 1 \leq \frac{d(m)^{3}}{n(n-1)(n-2)} .
$$

Thus, using the fact noted in Notation 2.1 that $d(m) \leq c_{\delta} m^{\delta}$,

$$
\begin{aligned}
P_{0}(n, m) & \leq \frac{d(m)\left(m^{2 s}+3 d(m) m^{s}+d(m)^{2}\right)}{n(n-1)(n-2)} \\
& \leq \frac{d(m) m^{2 s}\left(1+3 c_{\delta} m^{\delta-s}+\left(c_{\delta} m^{\delta-s}\right)^{2}\right)}{n(n-1)(n-2)}<\frac{c^{\prime} d(m) m^{2 s}}{n^{3}} .
\end{aligned}
$$

To estimate $c^{\prime}$ note first that, for $n \geq 6, n(n-1)(n-2)>n^{3} / 2$. Thus if $n \geq 6$ then, for any $m \geq n$ we may take $c^{\prime}=2\left(1+3 c_{\delta}+c_{\delta}^{2}\right)$. If $m \geq c_{\delta}^{1 /(s-\delta)}$, then $c_{\delta} m^{\delta-s} \leq 1$ and so we may take $c^{\prime}=10$. Theorem 1.5 now follows since $d(m)=m^{o(1)}$. Now assume that $n \geq m^{s}+c n^{a}$ for some positive constants $c$ and $a$. By Lemma 2.3,

$$
P_{0}(n, m)=\frac{1}{n} \sum_{d \in D_{s}} P_{0}(n-d, m)
$$


For each $d \in D_{s}$ we have $m>n-d \geq n-m^{s} \geq c n^{a}$, and hence applying Theorem 1.5 (which we have just proved),

$$
P_{0}(n-d, m)<\frac{c^{\prime} d(m) m^{2 s}}{(n-d)^{3}} \leq \frac{c^{\prime} d(m) m^{2 s}}{c^{3} n^{3 a}} .
$$

Thus, $P_{0}(n, m) \leq \frac{d(m)}{n}\left(\frac{c^{\prime} d(m) m^{2 s}}{c^{3} n^{3 a}}\right) \leq \frac{c^{\prime} c_{\delta}^{2} m^{2 s+2 \delta}}{c^{3} n^{1+3 a}}$.

\section{Proof of Theorem 1.1}

First we determine the 'very large' divisors of $m$ that are at most $n$.

Lemma 3.1 Let $r, m$ and $n$ be positive integers such that $r n \leq m<(r+1) n$.

(a) If $d$ is a divisor of $m$ such that $d \leq n$, then one of the following holds:

(i) $d=n=\frac{m}{r}$,

(ii) $d=\frac{m}{r+1}$ so that $\frac{r}{r+1} n \leq d<n$,

(iii) $d \leq \frac{m}{r+2}<\frac{r+1}{r+2} n$.

(b) Moreover, if $d_{1}, d_{2}$ are divisors of $m$ for which

$$
d_{1} \leq d_{2} \leq \frac{m}{r+1} \quad \text { and } \quad n \geq d_{1}+d_{2}>\frac{m(2 r+3)}{2(r+1)(r+2)},
$$

then $d_{1}=\frac{m}{c_{1}}, d_{2}=\frac{m}{c_{2}}$, where $c_{1}, c_{2}$ divide $m$, and satisfy $c_{2} \leq 2 r+3$, and either $r+2 \leq c_{2} \leq c_{1}<2(r+1)(r+2)$, or $c_{2}=r+1, c_{1} \geq r(r+1)$.

Proof As $d$ is a divisor of $m$ there is a positive integer $t$ such that $d=\frac{m}{t}$. Now $\frac{m}{t} \leq n \leq \frac{m}{r}$ and therefore $r \leq t$. If $r=t$ then $r$ divides $m$ and $d=\frac{m}{r} \leq n$, and since also $r n \leq m$ it follows that $d=\frac{m}{r}=n$ and (i) holds. If $t \geq r+2$ then (iii) holds. Finally, if $t=r+1$, then $d=\frac{m}{r+1}$ and $\frac{r}{r+1} n \leq \frac{m}{r+1}<n$ and hence (ii) holds.

Now we prove the last assertion. Suppose that $d_{1}, d_{2}$ are divisors of $m$ which are at most $\frac{m}{r+1}$, and such that $d_{1} \leq d_{2}$ and $n \geq d_{1}+d_{2}>\frac{m(2 r+3)}{2(r+1)(r+2)}$. Then, as $d_{1}$, $d_{2}$ divide $m$, there are integers $c_{1}, c_{2}$ such that $d_{1}=m / c_{1}$ and $d_{2}=m / c_{2}$. Since $d_{i} \leq m /(r+1)$ we have $c_{i} \geq r+1$ for $i=1,2$, and since $d_{1} \leq d_{2}$ we have $c_{1} \geq c_{2}$. Now $m / r \geq n \geq d_{1}+d_{2}>\frac{m(2 r+3)}{2(r+1)(r+2)}$, and hence $1 / r \geq 1 / c_{1}+1 / c_{2}>\frac{2 r+3}{2(r+1)(r+2)}$. If $c_{2} \geq 2(r+2)$ then, as $c_{1} \geq c_{2}$, we would have $1 / c_{1}+1 / c_{2} \leq 1 /(r+2)$, which is not the case. Thus $r+1 \leq c_{2} \leq 2 r+3$. If $c_{2} \geq r+2$, then

$$
\frac{1}{c_{1}}>\frac{2 r+3}{2(r+1)(r+2)}-\frac{1}{c_{2}} \geq \frac{2 r+3}{2(r+1)(r+2)}-\frac{1}{r+2}=\frac{1}{2(r+1)(r+2)}
$$

and hence $c_{1}<2(r+1)(r+2)$ as in the statement. On the other hand, if $c_{2}=r+1$, then

$$
\frac{1}{c_{1}} \leq \frac{n}{m}-\frac{1}{c_{2}} \leq \frac{1}{r}-\frac{1}{r+1}=\frac{1}{r(r+1)}
$$

so $c_{1} \geq r(r+1)$. 
The next result gives our first estimate of an upper bound for the proportion $P(n, m)$ of elements in $S_{n}$ of order dividing $m$. Recall our observation that the parameter $\delta$ in Notation 2.1(c) can be any positive real number; in Proposition 3.3 we will restrict to $\delta \leq s-\frac{1}{2}$. Note that the requirement $r n \leq m<(r+1) n$ implies that $\frac{n}{r+1} \leq n-\frac{m}{r+1} \leq \frac{m}{r(r+1)}$; the first case of Definition 3.2(b) below requires an upper bound of approximately half this quantity.

Definition 3.2 Let $r, m, n$ be positive integers such that $r n \leq m<(r+1) n$. Let $1 / 2<s \leq 3 / 4$ and $0<\delta \leq s-\frac{1}{2}$.

(a) Let $\alpha= \begin{cases}1 & \text { if } m=r n, \\ 0 & \text { otherwise. }\end{cases}$

(b) Let $\alpha^{\prime}= \begin{cases}1 & \text { if }(r+1) \text { divides } m \text { and } n-\frac{m}{r+1}<\frac{m}{2(r+1)(r+2)-1} \\ 0 & \text { otherwise. }\end{cases}$

(c) Let $t(r, m, n)$ denote the number of divisors $d$ of $m$ with $\frac{m}{2 r+3} \leq d \leq \frac{m}{r+1}$ such that there exists a divisor $d_{0}$ of $m$ satisfying

(i) $d+d_{0} \leq n$ and

(ii) $\frac{m}{2(r+1)(r+2)}<d_{0} \leq d$.

(d) Let $k(r, m, n)=t(r, m, n) \frac{2(r+1)(r+2)(2 r+3)}{r^{2}}$.

Proposition 3.3 Let $r, m, n, s$ and $\delta$ be as in Definition 3.2. Then, for a fixed value of $r$ and sufficiently large $n$,

$$
P(n, m) \leq \frac{\alpha}{n}+\frac{\alpha^{\prime} \cdot(r+1)}{m}+\frac{k(r, m, n)}{n^{2}}+O\left(\frac{1}{n^{1+2 s-2 \delta}}\right),
$$

where $\alpha, \alpha^{\prime}, t(r, m, n)$ and $k(r, m, n)$ are as in Definition 3.2. Moreover, $t(r, m, n) \leq$ $r+3$ and $k(r, m, n) \leq \frac{4(r+3)^{4}}{r^{2}}$.

Remark 3.4 (a) The term $\frac{1}{n}$, which occurs if and only if $m=r n$, corresponds to the $n$-cycles in $S_{n}$, and is the exact proportion of these elements. We refine the estimate for $P(n, r n)$ in Theorem 3.6 below.

(b) The term $\frac{r+1}{m}$, which occurs only if $r+1$ divides $m$ and $n-\frac{m}{r+1}<\frac{m}{2(r+1)(r+2)}$, corresponds to permutations with order dividing $m$ and having either one or two $s$-large cycles, with one (the larger in the case of two cycles) of length $\frac{m}{r+1}$. The proportion of elements of $S_{n}$ containing a cycle of length $\frac{m}{r+1}$ is $\frac{r+1}{m}$, and if there exists a positive integer $d \leq n-\frac{m}{r+1}$ such that $d$ does not divide $m$, then some of these elements have a $d$-cycle and hence do not have order dividing $m$. Thus $\frac{r+1}{m}$ may be an over-estimate for the proportion of elements in $S_{n}$ (where $n-\frac{m}{r+1}<\frac{m}{2(r+1)(r+2)}$ ) having order dividing $m$, having exactly one $s$-large cycle of length $\frac{m}{r+1}$, and possibly one additional $s$-large cycle of length dividing $m$. However it is difficult to make a more precise estimate for this term that holds for all sufficiently large $m, n$. In Theorem 3.6 we treat some special cases where this term either does not arise, or can be determined precisely.

(c) The term $\frac{k(r, m, n)}{n^{2}}$ arises as follows from permutations that have exactly two $s$-large cycles of lengths dividing $m$. For each of the $t(r, m, n)$ divisors $d$ of $m$ 
as in Definition 3.2(c), let $d_{0}(d)$ be the largest of the divisors $d_{0}$ satisfying Definition 3.2(c)(i),(ii). Note that $d_{0}(d)$ depends on $d$. Then $k(r, m, n) / n^{2}$ is an upper bound for the proportion of permutations of order dividing $m$ and having two $s$-large cycles of lengths $d$ and $d_{0}(d)$, for some $d$ satisfying $\frac{m}{2 r+3} \leq d \leq \frac{m}{r+1}$. As in (b) this term may be an over-estimate, not only for the reason given there, but also because lower bounds for the cycle lengths $d, d_{0}(d)$ were used to define $k(r, m, n)$. Indeed in the case $m=r n$ we are able to obtain the exact value of the coefficient of the $\frac{1}{n^{2}}$ summand.

Proof We divide the estimation of $P(n, m)$ into five subcases. Recall that, by (2), $P(n, m)$ is the sum of $P_{\geq 4}(n, m)$ and the $P_{i}(n, m)$, for $i=0,1,2,3$, where these are as defined in Notation 2.1. We will use the recursive formulae for $P_{\geq 4}(n, m)$ and the $P_{i}(n, m)$ in Lemma 2.3, together with the expressions for $P_{0}(n, m)$ in Theorem 1.5 and Lemma 2.4, to estimate these five quantities. Summing these estimates will give, by (2), our estimate for $P(n, m)$. We also use the information about divisors of $m$ in Lemma 3.1.

First we deal with $P_{0}(n, m)$. Since $r$ is fixed, it follows that, for sufficiently large $n$ (and hence sufficiently large $m$ ), we have $m^{s} \leq \frac{m}{r+2}$, which is less than $\frac{(r+1) n}{r+2}=$ $n-\frac{n}{r+2}$. Thus $n>m^{s}+\frac{n}{r+2}$, and applying Lemma 2.4 with $a=1, c=\frac{1}{r+2}$, it follows that

$$
P_{0}(n, m)=O\left(\frac{m^{2 s+2 \delta}}{n^{4}}\right)=O\left(\frac{1}{n^{4-2 s-2 \delta}}\right) \leq O\left(\frac{1}{n^{1+2 s-2 \delta}}\right)
$$

since $4-2 s-2 \delta \geq 1+2 s-2 \delta$ when $s \leq 3 / 4$.

Next we estimate $P_{3}(n, m)$ and $P_{\geq 4}(n, m)$. By Lemma 2.3 , the latter satisfies $P_{\geq 4}(n, m) \leq \frac{1}{24} \sum \frac{1}{d_{1} d_{2} d_{3} d_{4}}$, where the summation is over all ordered 4-tuples of $s$-large divisors of $m$ whose sum is at most $n$. Thus $P_{\geq 4}(n, m) \leq \frac{1}{24} \frac{d(m)^{4}}{m^{4 s}}=$ $O\left(\frac{1}{n^{4 s-4 \delta}}\right)$. Also

$$
P_{3}(n, m)=\frac{1}{6} \sum \frac{1}{d_{1} d_{2} d_{3}} P_{0}\left(n-d_{1}-d_{2}-d_{3}, m\right),
$$

where the summation is over all ordered triples of $s$-large divisors of $m$ whose sum is at most $n$. For such a triple $\left(d_{1}, d_{2}, d_{3}\right)$, if each $d_{i} \leq \frac{m}{4(r+1)}$, then $n-\sum d_{i} \geq$ $n-\frac{3 m}{4(r+1)}>\frac{n}{4}$, and so by Lemma $2.4, P_{0}\left(n-\sum d_{i}, m\right)=O\left(\frac{m^{2 s+\delta}}{n^{3}}\right)$. Thus the contribution of triples of this type to $P_{3}(n, m)$ is at most $O\left(\frac{d(m)^{3} m^{2 s+\delta}}{m^{3 s} n^{3}}\right)=O\left(\frac{1}{n^{3+s-4 \delta}}\right)$. For each of the remaining triples, the maximum $d_{i}$ is greater than $\frac{m}{4(r+1)}$ and in particular there is a bounded number of choices for the maximum $d_{i}$. Thus the contribution of the remaining triples to $P_{3}(n, m)$ is at most $O\left(\frac{d(m)^{2}}{m^{1+2 s}}\right)=O\left(\frac{1}{n^{1+2 s-2 \delta}}\right)$. It follows that

$$
P_{3}(n, m)+P_{\geq 4}(n, m)=O\left(\frac{1}{n^{x_{3}}}\right),
$$

where $x_{3}=\min \{4 s-4 \delta, 3+s-4 \delta, 1+2 s-2 \delta\}=1+2 s-2 \delta$ (using the fact that $\left.\delta \leq s-\frac{1}{2} \leq \frac{1}{4}\right)$. 
Now we estimate $P_{2}(n, m)$. By Lemma 2.3,

$$
P_{2}(n, m)=\frac{1}{2} \sum \frac{1}{d_{1} d_{2}} P_{0}\left(n-d_{1}-d_{2}, m\right),
$$

where the summation is over all ordered pairs of $s$-large divisors of $m$ whose sum is at most $n$. We divide these pairs $\left(d_{1}, d_{2}\right)$ into two subsets. The first subset consists of those for which $n-d_{1}-d_{2} \geq n^{v}$, where $v=(1+2 s+\delta) / 3$. Note that $v<1$ since $v \leq s+\frac{1}{6}<1$ (because $\delta \leq s-\frac{1}{2}$ and $\left.s \leq \frac{3}{4}\right)$. For a pair $\left(d_{1}, d_{2}\right)$ such that $n-d_{1}-d_{2} \geq n^{v}$, by Lemma $2.4, P_{0}\left(n-d_{1}-d_{2}, m\right)=O\left(\frac{m^{2 s+\delta}}{n^{3 v}}\right)$. Thus the total contribution to $P_{2}(n, m)$ from pairs of this type is at most $O\left(\frac{d(m)^{2} m^{2 s+\delta}}{m^{2 s} n^{3 v}}\right)=O\left(\frac{1}{n^{3 v-3 \delta}}\right)=$ $O\left(\frac{1}{n^{1+2 s-2 \delta}}\right)$.

Now consider pairs $\left(d_{1}, d_{2}\right)$ such that $n-d_{1}-d_{2}<n^{\nu}$. Since each $d_{i}<n \leq m / r$, it follows that each $d_{i} \leq m /(r+1)$. Since $v<1$, for sufficiently large $n$ (and hence sufficiently large $m$ ) we have $n^{v} \leq\left(\frac{m}{r}\right)^{v}<\frac{m}{2(r+1)(r+2)}$. Thus, for each of the pairs $\left(d_{1}, d_{2}\right)$ such that $n-d_{1}-d_{2}<n^{\nu}$, we have $d_{1}+d_{2}>n-n^{\nu}>\frac{m}{r+1}-\frac{m}{2(r+1)(r+2)}=$ $\frac{m(2 r+3)}{2(r+1)(r+2)}$, and hence one of $\left(d_{1}, d_{2}\right),\left(d_{2}, d_{1}\right)$ (or both if $\left.d_{1}=d_{2}\right)$ satisfies the conditions of Lemma 3.1(b). Thus, by Lemma 3.1(b), it follows that if $d_{1} \leq d_{2}$, then either $\left(d_{0}, d\right):=\left(d_{1}, d_{2}\right)$ satisfies the conditions of Definition 3.2(c), or $d_{2}=\frac{m}{r+1}$ and $d_{1} \leq \frac{m}{2(r+1)(r+2)}$. Let $P_{2}^{\prime}(n, m)$ denote the contribution to $P_{2}(n, m)$ from all the pairs $\left(d_{1}, d_{2}\right)$ where $\left\{d_{1}, d_{2}\right\}=\left\{\frac{m}{r+1}, d_{0}\right\}$ and $d_{0} \leq \frac{m}{2(r+1)(r+2)}$.

For the other pairs, we note that there are $t(r, m, n) \leq r+3$ choices for the larger divisor $d$. Consider a fixed $d \leq \frac{m}{r+1}$, say $d=\frac{m}{c}$. Then each divisor $d_{0}$ of $m$, such that $\frac{m}{2(r+1)(r+2)}<d_{0} \leq d$ and $d+d_{0} \leq n$, is equal to $\frac{m}{c_{0}}$ for some $c_{0}$ such that $c \leq c_{0}<2(r+1)(r+2)$. Let $d_{0}(d)=\frac{m}{c_{0}}$ be the largest of these divisors $d_{0}$. By Lemma 3.1(b), the combined contribution to $P_{2}(n, m)$ from the ordered pairs $\left(d, d_{0}(d)\right)$ and $\left(d_{0}(d), d\right)$ is (since $d$ and $d_{0}(d)$ may be equal) at most

$$
\frac{1}{d d_{0}(d)}<\frac{2 r+3}{m} \cdot \frac{2(r+1)(r+2)}{m}=\frac{2(r+1)(r+2)(2 r+3)}{m^{2}} .
$$

(Note that $\frac{1}{d d_{0}(d)} \geq \frac{(r+1)^{2}}{m^{2}}>\frac{1}{n^{2}}$.) If $d_{0}=\frac{m}{c^{\prime}}$ is any other divisor of this type and $d_{0}<d_{0}(d)$, then $c_{0}+1 \leq c^{\prime}<2(r+1)(r+2)$, and so $n-d-d_{0}=(n-d-$ $\left.d_{0}(d)\right)+d_{0}(d)-d_{0}$ is at least

$$
d_{0}(d)-d_{0}=\frac{m}{c_{0}}-\frac{m}{c^{\prime}} \geq \frac{m}{c_{0}}-\frac{m}{c_{0}+1}=\frac{m}{c_{0}\left(c_{0}+1\right)}>\frac{m}{4(r+1)^{2}(r+2)^{2}} .
$$

By Lemma 2.4 , the contribution to $P_{2}(n, m)$ from the pairs $\left(d, d_{0}\right)$ and $\left(d_{0}, d\right)$ is $O\left(\frac{1}{m^{2}} \cdot \frac{m^{2 s+\delta}}{m^{3}}\right)=O\left(\frac{1}{n^{5-2 s-\delta}}\right)$. Since there are $t(r, m, n) \leq r+3$ choices for $d$, and a bounded number of divisors $d_{0}$ for a given $d$, the contribution to $P_{2}(n, m)$ from all the pairs $\left(d_{1}, d_{2}\right)$ such that $n-d_{1}-d_{2}<n^{v}$ is at most

$$
P_{2}^{\prime}(n, m)+t(r, m, n) \frac{2(r+1)(r+2)(2 r+3)}{n^{2} r^{2}}+O\left(\frac{1}{n^{5-2 s-\delta}}\right),
$$


with $P_{2}^{\prime}(n, m)$ as defined above. Thus

$$
\begin{aligned}
P_{2}(n, m) & \leq P_{2}^{\prime}(n, m)+\frac{2 t(r, m, n)(r+1)(r+2)(2 r+3)}{n^{2} r^{2}}+O\left(\frac{1}{n^{x_{2}}}\right) \\
& =P_{2}^{\prime}(n, m)+\frac{k(r, m, n)}{n^{2}}+O\left(\frac{1}{n^{x_{2}}}\right)
\end{aligned}
$$

with $x_{2}=\min \{1+2 s-2 \delta, 5-2 s-\delta\}=1+2 s-2 \delta$. Note that

$$
k(r, m, n) \leq(r+3) \frac{2(r+1)(r+2)(2 r+3)}{r^{2}}=4 r^{2}+30 r+80+\frac{90}{r}+\frac{36}{r^{2}}
$$

which is less than $\frac{4(r+3)^{4}}{r^{2}}$.

Finally we estimate $P_{1}(n, m)+P_{2}^{\prime}(n, m)$. By Lemma 2.3, $P_{1}(n, m)=$ $\sum \frac{1}{d} P_{0}(n-d, m)$, where the summation is over all $s$-large divisors $d$ of $m$ such that $d \leq n$, and we take $P_{0}(0, m)=1$. Note that $d \leq n \leq \frac{m}{r}$, so each divisor $d=\frac{m}{c}$ for some $c \geq r$. In the case where $m=r n$, that is, the case where $n$ divides $m$ (and only in this case), we have a contribution to $P_{1}(n, m)$ of $\frac{1}{n}$ due to $n$-cycles. If $d<n$ then $d=\frac{m}{c}$ with $c \geq r+1$.

Next we consider all divisors $d$ of $m$ such that $d \leq \frac{m}{r+2}$. For each of these divisors, $n-d \geq n-\frac{m}{r+2} \geq n-\frac{(r+1) n}{r+2}=\frac{n}{r+2}$. Thus by Lemma 2.4, $P_{0}(n-d, m)=$ $O\left(\frac{m^{2 s+\delta}}{n^{3}}\right)=O\left(\frac{1}{n^{3-2 s-\delta}}\right)$. The number of $d$ satisfying $d \geq \frac{m}{2(r+1)}$ is bounded in terms of $r$ (which is fixed), and hence the contribution to $P_{1}(n, m)$ from all the divisors $d$ satisfying $\frac{m}{2(r+1)} \leq d \leq \frac{m}{r+2}$ is at most $O\left(\frac{1}{m} \frac{1}{n^{3-2 s-\delta}}\right)=O\left(\frac{1}{n^{4-2 s-\delta}}\right)$. On the other hand, if $m^{s} \leq d<\frac{m}{2(r+1)}$, then $n-d>n-\frac{(r+1) n}{2(r+1)}=\frac{n}{2}$. Now since $r$ is fixed and $s<1$, for sufficiently large $n$, we have $m^{s}<\frac{n}{4}$, and so $n-d>$ $m^{s}+\frac{n}{4}$. Then, by Lemma 2.4 (applied with $a=1$ and $c=\frac{1}{4}$ ), $P_{0}(n-d, m)=$ $O\left(\frac{m^{2 s+2 \delta}}{(n-d)^{4}}\right)=O\left(\frac{1}{n^{4-2 s-2 \delta}}\right)$, and the contribution to $P_{1}(n, m)$ from all $s$-large divisors $d<\frac{m}{2(r+1)}$ is at most $\frac{d(m)}{m^{s}} O\left(\frac{1}{n^{4-2 s-2 \delta}}\right)=O\left(\frac{1}{n^{4-s-3 \delta}}\right)$. Thus, noting that $\min \{4-2 s-\delta, 4-s-3 \delta\} \geq 1+2 s-2 \delta$, the contribution to $P_{1}(n, m)$ from all $s$-large divisors $d$ of $m$ such that $d \leq \frac{m}{r+2}$ is $O\left(\frac{1}{n^{1+2 s-2 \delta}}\right)$.

By Lemma 3.1, the only divisor not yet considered is $d=\frac{m}{r+1}$ and this case of course arises only when $r+1$ divides $m$. Suppose then that $r+1$ divides $m$. We must estimate the contribution to $P_{1}(n, m)+P_{2}^{\prime}(n, m)$ from elements containing a cycle of length $d=\frac{m}{r+1}$. The contribution to $P_{1}(n, m)+P_{2}^{\prime}(n, m)$ due to the divisor $d=\frac{m}{r+1}$ is $\frac{r+1}{m} P_{0}\left(n-\frac{m}{r+1}, m\right)+\frac{r+1}{m} \sum_{d_{0}} \frac{1}{d_{0}} P_{0}\left(n-\frac{m}{r+1}-d_{0}, m\right)$, where the summation is over all $s$-large $d_{0} \leq \frac{m}{2(r+1)(r+2)}$. Suppose first that $n-\frac{m}{r+1} \geq \frac{m}{2(r+1)(r+2)-1}$, so that for each $d_{0}, n-\frac{m}{r+1}-d_{0}>\frac{m}{2(r+1)^{2}(r+2)^{2}}$. Then, by Lemma 2.4, the contribution to $P_{1}(n, m)+P_{2}^{\prime}(n, m)$ is at most

$$
O\left(\frac{1}{m} \cdot \frac{m^{2 s+\delta}}{m^{3}}\right)+d(m) O\left(\frac{1}{m^{1+s}} \cdot \frac{m^{2 s+\delta}}{m^{3}}\right)=O\left(\frac{1}{n^{4-2 s-\delta}}\right)
$$

and this is $O\left(\frac{1}{n^{1+2 s-2 \delta}}\right)$ since $4-2 s-\delta \geq 1+2 s-2 \delta$. Finally suppose that $n-$ $\frac{m}{r+1}<\frac{m}{2(r+1)(r+2)}$. In this case we estimate the contribution to $P_{1}(n, m)+P_{2}^{\prime}(n, m)$ 
from $d=\frac{m}{r+1}$ by the proportion $\frac{1}{d}=\frac{r+1}{m}$ of elements of $S_{n}$ containing a $d$-cycle (recognising that this is usually an over-estimate). Putting these estimates together we have

$$
P_{1}(n, m)+P_{2}^{\prime}(n, m) \leq \frac{\alpha}{n}+\frac{\alpha^{\prime} \cdot(r+1)}{m}+O\left(\frac{1}{n^{1+2 s-2 \delta}}\right),
$$

where $\alpha=1$ if $m=r n$ and is 0 otherwise, and $\alpha^{\prime}=1$ if $r+1$ divides $m$ and $n-\frac{m}{r+1}<$ $\frac{m}{2(r+1)(r+2)-1}$, and is 0 otherwise. The result now follows using (2) and the estimates we have obtained for each of the summands.

It is sometimes useful to separate out the results of Proposition 3.3 according to the values of $m, n$. We do this in the theorem below, and also obtain in parts (a) and (b) exact asymptotic expressions for $P(n, r n)$ and $P(n, t !(n-t))$ where $r, t$ are bounded and $n$ is sufficiently large. For this it is convenient to define two sets of integer pairs.

Definition 3.5 For positive integers $r$ and $m$, define the following sets of integer pairs:

$$
\mathcal{T}(r)=\left\{(i, j) \mid 1 \leq i, j \leq r^{2}, i j=r^{2}, \text { and both } r+i, r+j \text { divide } m\right\}
$$

and $\mathcal{T}^{\prime}(r)=\left\{(i, j) \mid 1<i, j \leq(r+1)^{2},(i-1)(j-1)=(r+1)^{2}\right.$, and both $r+i$, $r+j$ divide $m\}$.

Theorem 3.6 Let $n, m, r$ be positive integers such that $r n \leq m<(r+1) n$. Let $1 / 2<$ $s \leq 3 / 4$ and $0<\delta \leq s-1 / 2$. Then, the following hold for $r$ fixed and sufficiently large $n$ (where the sets $\mathcal{T}(r)$ and $\mathcal{T}^{\prime}(r)$ are as in Definition 3.5).

(a) If $m=r n$, then $P(n, m)=\frac{1}{n}+\frac{c(r)}{n^{2}}+O\left(\frac{1}{n^{1+2 s-2 \delta}}\right)$, where $c(r)=\sum_{(i, j) \in \mathcal{T}(r)}\left(1+\frac{i+j}{2 r}\right)$. In particular $c(1)=0$ if $n$ is odd, and 2 if $n$ is even.

(b) If $r=t$ ! -1 and $m=t$ ! $(n-t)=(r+1) n-t \cdot t$ !, then

$$
\begin{aligned}
& P(n, m)=\frac{1}{n-t}+\frac{c^{\prime}(r)}{(n-t)^{2}}+O\left(\frac{1}{n^{1+2 s-2 \delta}}\right), \text { where } \\
& c^{\prime}(r)=\sum_{(i, j) \in \mathcal{T}^{\prime}(r)}\left(1+\frac{i+j-2}{2(r+1)}\right) .
\end{aligned}
$$

(c) If $r n<m$, then $P(n, m) \leq \frac{\alpha^{\prime} .(r+1)}{m}+\frac{k(r, m, n)}{n^{2}}+O\left(\frac{1}{n^{1+2 s-2 \delta}}\right)$, where $\alpha^{\prime}$ and $k(r, m, n)$ are as in Definition 3.2 .

Proof Part (c) follows immediately from Proposition 3.3. Next we prove part (a). Suppose that $m=r n$. If $r+1$ divides $m$ then we have $n-\frac{m}{r+1}=\frac{m}{r(r+1)}>$ $\frac{m}{2(r+1)(r+2)-1}$. It follows from Proposition 3.3 that $P(n, m) \leq \frac{1}{n}+\frac{k(r, m, n)}{n^{2}}+$ $O\left(\frac{1}{n^{1+2 s-2 \delta}}\right)$. To complete the proof we refine the argument given in the proof of 
Proposition 3.3 for $P_{2}(n, m)$ which gave rise to the term $\frac{k(r, m, n)}{n^{2}}$. The elements contributing to this term were those with exactly two $s$-large cycles, where one of these cycles had length $d=\frac{m}{r+i}$ for some $i$ such that $1 \leq i \leq r+3$, and the other had length $d_{0}(d)=\frac{m}{r+j}$ for some $j$ such that $r+i \leq r+j<2(r+1)(r+2)$ and $d+d_{0}(d) \leq n$. Moreover, for a given value of $d$, the value of $d_{0}(d)$ was the largest integer with these properties. Since we now assume that $m=r n$ we have

$$
d+d_{0}(d)=\frac{m(2 r+i+j)}{(r+i)(r+j)} \leq n=\frac{m}{r}
$$

that is, $r(2 r+i+j) \leq(r+i)(r+j)$, which is equivalent to $r^{2} \leq i j$. If $d+d_{0}(d)$ is strictly less than $n$, that is to say, if $r^{2}<i j$, and thus $i j-r^{2} \geq 1$, then

$$
n-d-d_{0}(d)=n-\frac{r n(2 r+i+j)}{(r+i)(r+j)}=\frac{n\left(i j-r^{2}\right)}{(r+i)(r+j)} \geq \frac{n}{(r+i)(r+j)},
$$

and since $i \leq r+3$ and $r+j<2(r+1)(r+2)$ we have $\frac{n}{(r+i)(r+j)} \geq \frac{n}{2(r+1)(r+2)(2 r+3)}$.

It now follows from Lemma 2.4 that the contribution to $P_{2}(n, m)$ from all ordered pairs $\left(d, d_{0}(d)\right)$ and $\left(d_{0}(d), d\right)$ with $d, d_{0}(d)$ as above and $n>d+d_{0}(d)$ is $O\left(\frac{1}{n^{2}} \frac{m^{2 s+\delta}}{n^{3}}\right)=O\left(\frac{1}{n^{5-2 s-\delta}}\right) \leq O\left(\frac{1}{n^{1+2 s-2 \delta}}\right)$. Thus when $m=r n$, the only contributions to the $O\left(\frac{1}{n^{2}}\right)$ term come from pairs $\left(\frac{m}{r+i}, \frac{m}{r+j}\right)$ such that $r^{2}=i j$ and $1 \leq i, j \leq r^{2}$. (Note that we no longer assume $i \leq j$.) These are precisely the pairs $(i, j) \in \mathcal{T}(r)$. For such a pair $\left(\frac{m}{r+i}, \frac{m}{r+j}\right)$, the contribution to $P_{2}(n, m)$ is

$$
\frac{1}{2} \cdot \frac{r+i}{m} \cdot \frac{r+j}{m}=\frac{r^{2}+r(i+j)+i j}{2 n^{2} r^{2}}=\frac{1}{n^{2}}\left(1+\frac{i+j}{2 r}\right)
$$

(since $\left.i j=r^{2}\right)$. Thus $P(n, m) \leq \frac{1}{n}+\frac{c(r)}{n^{2}}+O\left(\frac{1}{n^{1+2 s-2 \delta}}\right)$. Moreover, for each $(i, j) \in \mathcal{T}(r)$, each permutation in $S_{n}$ having exactly two cycles of lengths $\frac{m}{r+i}$ and $\frac{m}{r+j}$ is a permutation of order dividing $m$. Thus $P(n, r n) \geq \frac{1}{n}+\frac{c(r)}{n^{2}}$, and the main assertion of part (a) is proved. Finally we note that, if $r=1$ then the only possible pair in $\mathcal{T}(1)$ is $(1,1)$, and for this pair to lie in the set we require that $r+1=2$ divides $m=n$. Thus $c(1)$ is 0 if $n$ is odd, and is 2 if $n$ is even.

Finally we prove part (b) where we have $r=t !-1$ and $m=t !(n-t)$. Then $r n=$ $(t !-1) n=m+t \cdot t !-n$ which is less than $m$ if $n>t \cdot t$. Also $(r+1) n=t ! n>m$. Thus, for sufficiently large $n$, we have $r n<m<(r+1) n$. Moreover, $r+1$ divides $m$ and $n-\frac{m}{r+1}=n-(n-t)=t$, which for sufficiently large $n$ is less than $\frac{n-t}{3 t !}<$ $\frac{m}{2(r+1)(r+2)-1}$. It now follows from part (c) that $P(n, t !(n-t)) \leq \frac{1}{n-t}+\frac{k(r, m, n)}{n^{2}}+$ $O\left(\frac{1}{n^{1+2 s-2 \delta}}\right)$. Our next task is to improve the coefficient of the $O\left(\frac{1}{n^{2}}\right)$ term using a similar argument to the proof of part (a). The elements contributing to this term have exactly two $s$-large cycles of lengths $d=\frac{m}{r+i}$ and $d_{0}(d)=\frac{m}{r+j}$, with $r+i, r+j \leq$ $(r+1)(r+2)$ and

$$
d+d_{0}(d)=\frac{m(2 r+i+j)}{(r+i)(r+j)} \leq n=\frac{m}{r+1}+t .
$$


This is equivalent to $(r+1)(2 r+i+j) \leq(r+i)(r+j)+\frac{t(r+1)(r+i)(r+j)}{m}$, and hence, for sufficiently large $n$ (and hence sufficiently large $m),(r+1)(2 r+i+j) \leq$ $(r+i)(r+j)$. This is equivalent to $(i-1)(j-1) \geq(r+1)^{2}$. If $(i-1)(j-1)>$ $(r+1)^{2}$, then

$$
\begin{aligned}
n-d-d_{0}(d) & =\left(t+\frac{m}{r+1}\right)-\frac{m(2 r+i+j)}{(r+i)(r+j)} \\
& =t+\frac{m\left((i-1)(j-1)-(r+1)^{2}\right)}{(r+1)(r+i)(r+j)} \\
& >\frac{r n}{(r+1)^{3}(r+2)^{2}} .
\end{aligned}
$$

As for part (a), the contribution to $P_{2}(n, m)$ from all pairs $\left(\frac{m}{r+i}, \frac{m}{r+j}\right)$ with $(i-1)(j-1)>(r+1)^{2}$ is $O\left(\frac{1}{n^{1+2 s-2 \delta}}\right)$. Thus the only contributions to the $O\left(\frac{1}{n^{2}}\right)$ term come from pairs $\left(d, d_{0}(d)\right)=\left(\frac{m}{r+i}, \frac{m}{r+j}\right)$ such that $(r+1)^{2}=(i-1)(j-1)$ and $1 \leq i, j \leq(r+1)^{2}$. These are precisely the pairs $(i, j) \in \mathcal{T}^{\prime}(r)$. For each of these pairs we have $r^{2}+2 r=i j-i-j$ and the contribution to $P_{2}(n, m)$ is

$$
\begin{aligned}
\frac{1}{2 d d_{0}(d)} & =\frac{(r+i)(r+j)}{2 m^{2}}=\frac{r^{2}+r(i+j)+i j}{2(r+1)^{2}(n-t)^{2}} \\
& =\frac{(r+1)(2 r+i+j)}{2(r+1)^{2}(n-t)^{2}}=\frac{1}{(n-t)^{2}}\left(1+\frac{i+j-2}{2(r+1)}\right) .
\end{aligned}
$$

Thus $P(n, m) \leq \frac{1}{n-t}+\frac{c^{\prime}(r)}{(n-t)^{2}}+O\left(\frac{1}{n^{1+2 s-2 \delta}}\right)$. On the other hand, each permutation in $S_{n}$ that contains an $(n-t)$-cycle has order dividing $t !(n-t)=m$, and the proportion of these elements is $\frac{1}{n-t}$. Also, for each $(i, j) \in \mathcal{T}^{\prime}(r)$, each permutation in $S_{n}$ having exactly two cycles of lengths $\frac{m}{r+i}$ and $\frac{m}{r+j}$, and inducing any permutation on the remaining $n-\frac{m}{r+i}-\frac{m}{r+j}=t$ points, is a permutation of order dividing $m=t !(n-t)$, and the proportion of all such elements is $\frac{c^{\prime}(r)}{(n-t)^{2}}$. Thus $P(n, m) \geq \frac{1}{n-t}+\frac{c^{\prime}(r)}{(n-t)^{2}}$, and the assertion of part (b) is proved.

It is a simple matter now to prove Theorems 1.1 and 1.2.

Proof of Theorems 1.1 and 1.2 The first theorem follows from Theorem 3.6(a) and (b) on setting $s=3 / 4$ and allowing $\delta \rightarrow 0$. Note that $\frac{1}{n-t}=\frac{1}{n}+\frac{t}{n^{2}}+O\left(\frac{1}{n^{3}}\right)$ and $\frac{1}{(n-t)^{2}}=$ $\frac{1}{n^{2}}+O\left(\frac{1}{n^{3}}\right)$. For the second theorem, again we set $s=3 / 4$ in Theorem 3.6(c). By Proposition 3.3 we have $k(r, m, n) \leq \frac{4(r+3)^{4}}{r^{2}}$. If we define $k(r)=\frac{4(r+3)^{4}}{r^{2}}$ the result follows.

Finally we derive the conditional probabilities in Corollary 1.4.

Proof of Corollary 1.4 Let $r, n$ be positive integers with $r$ fixed and $n$ 'sufficiently large', and let $g$ be a uniformly distributed random element of $S_{n}$. First set $m=r n$. 
Let $A$ denote the event that $g$ is an $n$-cycle, and let $B$ denote the event that $g$ has order dividing $m$, so that the probability $\operatorname{Prob}(B)$ is $P(n, m)$. Then, by elementary probability theory, we have

$$
\operatorname{Prob}(A \mid B)=\frac{\operatorname{Prob}(A \cap B)}{\operatorname{Prob}(B)}=\frac{\operatorname{Prob}(A)}{\operatorname{Prob}(B)}=\frac{\frac{1}{n}}{P(n, m)} .
$$

By Theorem 1.1, $\frac{1}{n}+\frac{c(r)}{n^{2}}<P(n, m)=\frac{1}{n}+\frac{c(r)}{n^{2}}+O\left(\frac{1}{n^{2.5-o(1)}}\right)$, and hence

$$
1-\frac{c(r)}{n}-O\left(\frac{1}{n^{1.5-o(1)}}\right) \leq \operatorname{Prob}(A \mid B) \leq 1-\frac{c(r)}{n}+O\left(\frac{1}{n^{2}}\right) .
$$

Now suppose that $r=t !-1$ for some integer $t \geq 2$, and let $A$ denote the event that $g$ contains an $(n-t)$-cycle, so that $\operatorname{Prob}(A)=\frac{1}{n-t}$. Then, with $B$ as above for the integer $m:=t !(n-t)$, we have

$$
\operatorname{Prob}(A \mid B)=\frac{\operatorname{Prob}(A \cap B)}{\operatorname{Prob}(B)}=\frac{\operatorname{Prob}(A)}{\operatorname{Prob}(B)}=\frac{\frac{1}{n-t}}{P(n, m)} .
$$

By Theorem 3.6(b), $\frac{1}{n-t}+\frac{c^{\prime}(r)}{(n-t)^{2}}<P(n, m)=\frac{1}{n-t}+\frac{c^{\prime}(r)}{(n-t)^{2}}+O\left(\frac{1}{n^{2.5-o(1)}}\right)$, and hence

$$
1-\frac{c^{\prime}(r)}{n}-O\left(\frac{1}{n^{1.5-o(1)}}\right) \leq \operatorname{Prob}(A \mid B) \leq 1-\frac{c^{\prime}(r)}{n}+O\left(\frac{1}{n^{2}}\right)
$$

Acknowledgements This research was supported by ARC Discovery Grants DP0209706 and DP0557587. The authors thank the referee for reading the submitted version carefully and for valuable advice on the paper.

\section{References}

1. Beals, R., Leedham-Green, C. R., Niemeyer, A. C., Praeger, C. E., \& Seress, Á. (2003). A black-box group algorithm for recognizing finite symmetric and alternating groups. I. Trans. Am. Math. Soc. (electronic), 355(5), 2097-2113.

2. Bouwer, I. Z., \& Chernoff, W. W. (1985). Solutions to $x^{r}=\alpha$ in the symmetric group. In Tenth British Combinatorial conference (Glasgow, 1985). Ars Comb. (A), 20, 83-88.

3. Chowla, S., Herstein, I. N., \& Scott, W. R. (1952). The solutions of $x^{d}=1$ in symmetric groups. Norske Vid. Selsk., 25, 29-31.

4. Erdős, P., \& Turán, P. (1965). On some problems of a statistical group-theory. I. Wahrsch. Verw. Geb., 4, 175-186.

5. Erdős, P., \& Turán, P. (1967). On some problems of a statistical group-theory. III. Acta Math. Acad. Sci. Hung., 18, 309-320.

6. Gao, L., \& Zha, J. G. (1987). Solving the equation $x^{n}=\sigma$ in the symmetric group $S_{m}$. J. Math. (Wuhan), 7(2), 173-176.

7. Landau, E. (1909). Handbuch der Lehre von der Verteilung der Primzahlen. Leipzig: Teubner.

8. Mineev, M. P., \& Pavlov, A. I. (1976). An equation in permutations. Trudy Mat. Inst. Steklov., 142(270), 182-194.

9. Mineev, M. P., \& Pavlov, A. I. (1976). The number of permutations of a special form, Mat. Sbornik (N.S.), 99(141)(3), 468-476, 480.

10. Moser, L., \& Wyman, M. (1955). On solutions of $x^{d}=1$ in symmetric groups. Can. J. Math., 7, $159-168$.

11. Moser, L., \& Wyman, M. (1956). Asymptotic expansions. Can. J. Math., 8, 225-233. 
12. Niemeyer, A. C., \& Praeger, C. E. (2005). On the proportion of permutations of order a multiple of the degree. Preprint.

13. Niemeyer, A. C., \& Praeger, C. E. (2006). On the frequency of permutations containing a long cycle. J. Algebra, 300, 289-304.

14. Niven, I., Zuckerman, H. S., \& Montgomery, H. L. (1991). An introduction to the theory of numbers (5th ed.). New York: Wiley.

15. Volynets, L. M. (1986). The number of solutions of the equation $x^{s}=e$ in a symmetric group. Mat. Zametki, 40, 155-160, 286.

16. Warlimont, R. (1978). Über die Anzahl der Lösungen von $x^{n}=1$ in der symmetrischen Gruppe $S_{n}$. Arch. Math. (Basel), 30(6), 591-594.

17. Wilf, H. S. (1986). The asymptotics of $e^{P(z)}$ and the number of elements of each order in $S_{n}$. Bull. Am. Math. Soc. (N.S.), 15(2), 228-232. 